\title{
PROGRESS IN OPTOELECTRONIC OSCILLATORS
}

\author{
Nabin K. Raut*1, Jeffery Miller ${ }^{1}$, Jay Sharping ${ }^{1}$ \\ ${ }^{1}$ University of California, Merced, USA \\ *Corresponding author: nraut@ucmerced.edu
}

(Received: October 1, 2018; Revised: April 1, 2019; Accepted: April 12, 2019)

\begin{abstract}
An optoelectronic oscillator (OEO) generates a spectrally pure and ultra-stable radio frequency signal from a continuous wave laser source (Yao et al. 2004). In a conventional electrical oscillator, the energy storage capacity is limited, which compromises stability of the signal. To address this issue, Yao and Maleki invented the optoelectronic oscillator in 1996. This novel oscillator uses low-loss optical fiber to extend the length of the oscillator and thereby increases the amount of energy that can be stored (Madjar \& Tibor 2006). Due to this additional energy storing component in the system, the purity and stability of the signal increase significantly. Following their invention, many modifications have been made over the years to improve the frequency stability of OEOs (lower phase noise and timing jitter). This review article discusses some of those key developments and then introduces some ongoing work devoted to understanding the impact of using electrical filters with $\mathrm{Q}>10^{9}$.
\end{abstract}

Keywords: Dual loop OEO, Injection-locking OEO, Optoelectronic oscillator, Phase noise

\section{INTRODUCTION}

Stable electrical and optical frequency generation is central to communications and computation (Couch 1995). Data modulation and demodulation requires a stable carrier at the transmitter and a local oscillator at the receiver. Using a signal generator for Doppler radar demands a pure microwave signal (Hao et al. 2018, Maleki 2012). Similarly, telecommunication applications rely on highly stable clock signals (Salik \& Maleki 2007). The above applications drive research into signal generation stability improvements (Chen et al. 2011). One contribution to the stability of an oscillator is the energy loss. The search for a low-loss oscillator led to the invention of the optoelectronic oscillator (OEO) (Neyer \& Voges). The OEO takes advantage of the low loss, low dispersion, and high efficiency of optical fiber to transmit a modulated signal. Moreover, it has electrical and optical outputs that can be used as the basis for other devices or experiments. The low phase noise electrical signal can be utilized for applications of sensing and detection (e.g. radar) (Cui et al. 2018). On the optical side of the device, the low timing jitter, high precision clock signal can be used in high speed telecommunications, naval navigation, and clock recovery (Eliyahu et al. 2003, Vorreau 2010). In this review we describe the main components of an OEO and some of the most common OEO configurations including single and multi-loop OEOs. We then review how each configuration impacts the overall performance of the OEO system. Finally, we discuss how to improve the performance of OEO systems by using high qualityfactor $(\mathrm{Q})$ band pass filters.

Figure 1 (Yao \& Maleki 1996a) shows a simple diagram of an OEO. Continuous-wave light from a pump laser is passed through a fiber delay, optically amplified and detected, converting the optical signal into a microwave electrical signal. The electrical signal then passes through the band pass filter is amplified and fed back into the modulator. When the overall gain in the loop is greater than the loss, the system begins to oscillate at resonant frequencies which are determined by the length of the loop. The band pass filter selects one mode out of all competing modes under its bandwidth producing a spectrally-pure RF signal at the RF output.

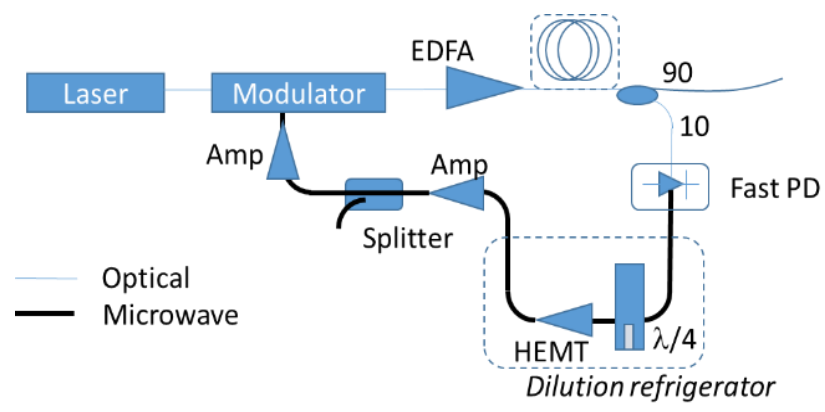

Fig. 1. A diagram of an OEO which includes a high-Q band pass filter in a dilution refrigerator. The heavy and thick lines optical and electrical connections, respectively. The system forms a feedback loop which oscillates when the gain is sufficient to compensate for the losses in the loop (Yao \& Maleki 1996a)

The hybrid electro-optical loop configuration and the band-pass filter are two crucial components of the OEO. The optical fiber allows the loop to be greater than $1 \mathrm{~km}$ in length and thus produces multiple closely-spaced spectral modes, about which will discuss in detail in subsequent paragraphs. The mode spacing is inversely proportional to the total length of the loop. The band pass filter favors a single mode. Figure 2 (Yao \& Maleki 1996a) 
illustrates the mode selection process of the OEO system. Multiple oscillator modes lie within the filter bandwidth. If we use filter with a $\mathrm{Q}$ on the order of ten thousand and a $3 \mathrm{~km}$-long fiber spool, 149 modes will be present under the band pass envelope. One of these modes is transmitted through the filter with the least loss and becomes the output mode, while the others are suppressed (Jiang et al. 2013).

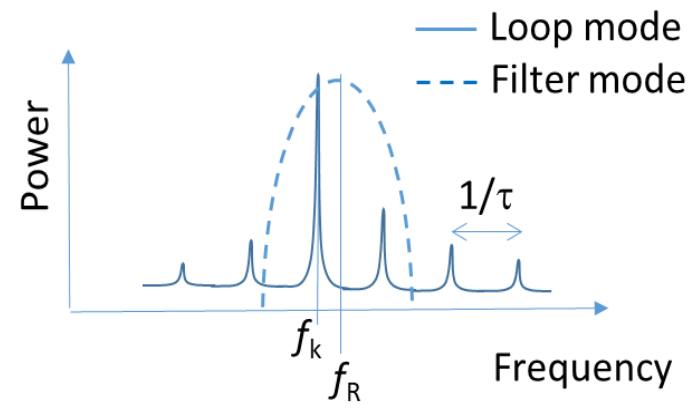

Fig. 2. Illustration of the filter response to the oscillator modes within the filter bandwidth. There can be large number of fiber modes within the filter bandwidth. The mode with the least loss will oscillate and the others will be suppressed ( ${ }^{1}$ Yao et al. 1996). Figure adapted with permission of The Optical Society

The overall length of the oscillator, and thus the oscillator mode spacing, is largely determined by the length of the optical fiber. For example, fiber lengths of 200 meters, 2 kilometers, and 3 kilometers equate to a mode spacings of $1 \mathrm{MHz}, 100 \mathrm{KHz}$, and $67 \mathrm{KHz}$, respectively. This proportionality is given by the relation $\Delta f=\frac{1}{\tau}=\frac{c}{n l}$

(Maleki 2012), where $n$ is refractive index of the material, $c$ is velocity of light and $l$ is the fiber delay length. The optical path length of the fiber can vary because of thermal expansion or contraction, nonlinear effects, or mechanical vibrations. Among all these effects, thermal contributions are the most detrimental. In fact, thermal fluctuations cause changes in both the physical length as well as the refractive index of the fiber, which in turn results in instability of the resonant frequency. The thermal dependence of the resonance frequency is given by $\frac{\Delta f}{\Delta T}=-\frac{f_{k}}{n} \times \frac{\Delta n}{\Delta T}$ (Eliyahu et al. 2003), where $f_{\mathrm{k}}$ is the position of a particular mode.

The Q of an oscillator can be defined in several ways. The most useful definition for our purposes is that $Q$ is the ratio of the energy stored in the oscillator to the energy lost per cycle. The long optical fiber in an OEO acts as an efficient energy storage component. The quality factor is given by $Q=2 \pi f_{k} \tau$ (Liu et al. 2018) and it depends on the OEO oscillation frequency $\left(f_{k}\right)$ selected by the band pass filter whose center frequency is $f_{R}$ and the total loop delay time $(\tau)$. The total loop delay time is the sum of the optical delay time $\left(\tau_{o p}\right)$, which is proportional to the length of the fiber, and the RF delay time $\left(\tau_{R F}\right)$, which is mainly due to amplifiers and the cavity (Romisch et al. 2000). The RF delay time is determined by relation $\tau_{R F}=\frac{\emptyset_{1}+\emptyset_{2}+\cdots+\emptyset_{n}}{2 \pi f_{k}}+2 Q_{L} \frac{f_{k}-f_{R}}{2 \pi f_{k} f_{R}}$ (Romisch \& De Marchi 1999). Where, $\emptyset_{1}, \emptyset_{2}, \ldots \emptyset_{n}$ are the phase shifts introduced by the amplifiers used in the OEO, $\mathrm{Q}_{\mathrm{L}}$ is the loaded quality factor of the band pass filter, $f_{k}$ is the OEO oscillation frequency cavity, and $f_{R}$ is the center angular frequency (Romisch \& De Marchi 1999). In practice, $\tau_{o p} \gg \tau_{R F}$, hence the optical delay plays the dominant role in determining Q. A longer fiber corresponds to a higher Q. For example, if we use $4 \mathrm{~km}$ of fiber we obtain $\mathrm{Q}$ on the order of $10^{6}$ (Merrer et al. 2008). Importantly, higher value of $\mathrm{Q}$ means substantial reduction of timing jitter, the phase noise analog in the time domain (Nelson et al. 2007).

One of the important figures of merit that measure quality of the OEOs is its phase noise performance. The lower the phase noise, the better the OEO. Phase noise is a measure of the amount of unwanted frequency variations about the nominal frequency (Rutman 1978). It is characterized by a one-sided spectral density referred to as the single side band (SSB) phase noise. The one-sided spectral density is the energy distribution as a continuous function per unit bandwidth, $\mathrm{L}(\mathrm{f})=\frac{\text { Area of } 1 \mathrm{HZ} \text { bandwidth }}{\text { Total area under the curve }}$ or $L_{\text {total }}\{\Delta f\}=10 \log \left[\frac{P_{\text {sideband }}\left(f_{k}+\Delta f_{i} 1 \mathrm{~Hz}\right)}{P_{\text {carrier }}}\right]$, Fig $3 b$.

Here, $P_{\text {sideband }}\left(f_{k}+\Delta f, 1 \mathrm{~Hz}\right)$ is single side-band power measured at an offset frequency of $\Delta f$ from the central frequency with $1 \mathrm{~Hz}$ bandwidth, and $\boldsymbol{P}_{\text {carrier }}$ is power of the carrier signal. It is given in units of $\mathrm{dBc} / \mathrm{Hz}$ (Hajimiri 1998). As shown in figure 3, the presence of small phase fluctuations in the input signal can add a significant amount of phase noise to the output signal and jitter in the clock signal. The ideal, noise-less and nondrifting, sinusoidal signal is represented by $x(t)=A(t) \operatorname{Sin}(2 \pi f t)$. This signal will show single resonant frequency in power spectral density (PSD) as shown in figure 3 a. In contrast, the noisy input signal with time dependent phase variation, $\phi(t)$, is given by $x(t)=A(t) \operatorname{Sin}(2 \pi f t+\phi(t)) \approx \operatorname{Sin}(2 \pi f t)+\operatorname{Sin}(\phi(t))$ and it will be less stable. In this case, energy is spread over range of frequencies offset from the central frequency, as shown in Fig. $3 b$. 
a.

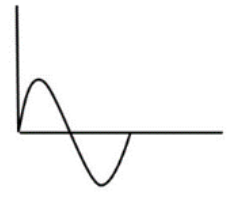

Ideal sinusoidal wave

b.

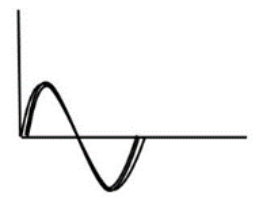

Noisy sinusoidal wave

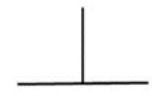

Output signal

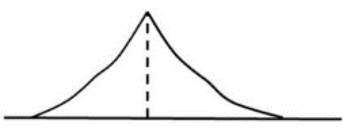

Output signal

Fig. 3: Schematic illustration of (a) the ideal sinusoidal signal and its power spectrum density (PSD). (b) In the presence of phase noise, instability in the signal is introduced. This instability can spread over large area around nomial frequency

Noise can come from many sources. Thermal, shot and flicker noise are intrinsic to the microwave devices being used, while environmental and power supply fluctuations are external sources of noise (Hajimiri 1998). The different sources of noise can be recognized by their locations at particular offset frequency ranges around the resonance frequency. For example, thermal and acoustic (environmental) vibrations dominate in the first few hertz offset from the carrier. Microwave amplifiers add flicker (also known as 1/f) noise (Romisch et al. 2000) to the system which shows up from few hertz to kilohertz. Optical amplifiers contribute to the noise by amplifying the non-oscillating side modes which show up beyond 10 $\mathrm{kHz}$ (Devgan 2013). Similarly, the optical to electrical detector is source of shot and thermal noise (Rubiola et al. 2006). Thermal noise also comes from thermal drifting of the laser oscillation frequency (Marshall et al. 2000), the temperature dependence of the refractive index of the optical fiber (Shelby et al. 1985), the stability of the electro-optic modulator (Yao \& Maleki 1996a), and thermal fluctuations in the gain of the amplifiers (Lesson 1966). In addition, the laser exhibits laser random intensity noise (RIN) (Marshall \& Yariv 2000). Power supply noise at $60 \mathrm{~Hz}$ is due to supply ac-source (Zhou \& Blasche 2005). Minimizing the noise requires choosing the best components and designing the system to reduce environmental and thermal issues (Eliyahu et al. 2003, Kaba et al. 2006, Romisch et al. 2000).

The band pass filter is a crucial component in the OEO as it selects a single mode from multiple input modes. Using high Q cavity as the band pass filter leads to higher energy storage in the system and greater stability in the signal. The equation $\frac{\Delta f}{f_{k}}=\frac{\Delta \varphi}{Q}$, shows the dependence of the conversion from phase uncertainty into frequency

uncertainty on the quality factor of the filter, where $\Delta f$ is the frequency uncertainty, $f_{k}$ is the resonant frequency, $\Delta \emptyset$ is the uncertainty in phase, $\mathrm{Q}$ is the quality factor of filter (Leeson 1966). A high-Q cavity will minimize conversion of source phase fluctuations into frequency fluctuations.

\section{Single loop OEO (SOEO)}

The schematic diagram of a single-loop OEO (SOEO) is shown in Fig. 1 (Yao \& Maleki 1996a). As the name suggests, in the single loop OEO the main loop starts and ends at the electro-absorption modulator with no branches in between except for measurements and outputs. The main optical components are a continuous-wave laser, an intensity modulator such as an electro-optic or electroabsorption modulator, an optical-fiber delay, and a fast (up to $110 \mathrm{GHz}$ ) photo-detector. The electrical components include a band pass filter with $\mathrm{Q}$ ranging from $10^{3}-10^{12}$, microwave amplifiers and microwave splitters (Yao \& Maleki 1996a). The oscillation frequency is selected from the available loop modes by the band pass filter according to the equation below:

$$
\begin{aligned}
& f_{o s c}=f_{k}=\frac{\left(k+\frac{1}{2}\right)}{\tau} \text { for } G\left(V_{o s c}\right)<0 \\
& f_{o s c}=f_{k}=\frac{k}{\tau} \text { for } G\left(V_{o s c}\right)>0
\end{aligned}
$$

Where, $k$ is the mode selected by the filter, $\tau$ is total energy decay time and $G\left(V_{\text {osc }}\right)$ is the gain coefficient, which can be made negative by using an inverting amplifier or by adjusting the bias of the amplitude modulator. If gain coefficient is negative the fundamental mode is $f_{0}=1 / 2 \tau$ meaning it takes two passes through the loop to obtain constructive interference. If the gain coefficient is positive the fundamental mode is $f_{0}=1 / \tau$ (Yao \& Maleki 1996a).

In the SOEO, most of the studies evaluate the stability as a function of the length of the fiber. The longest fiber used so far is by (Eliyahu et al. 2008). They used 16 kilometers fiber with help of a thermal stabilization mechanism. Most importantly, in almost all works in the SOEO, reduced phase noise was observed with increased fiber delay. Lasri et al. (2003) concluded that, as shown in figure 4, timing jitter decreases as the length of the fiber delay increases. They used five different fiber lengths: 400 meters, 1 kilometer, 1.7 kilometers, 2.4 kilometers, and 3 kilometers and found lowest jitter in the longest loop. However, thermal vibration precludes increasing the fiber length past a certain point (Yao \& Maleki 1996a). The lowest phase noise SOEO claim so far is by Lasri with -115 $\mathrm{dBc} / \mathrm{Hz}$ phase noise at a $10 \mathrm{KHz}$ offset frequency (Lasri et al. 2003). 


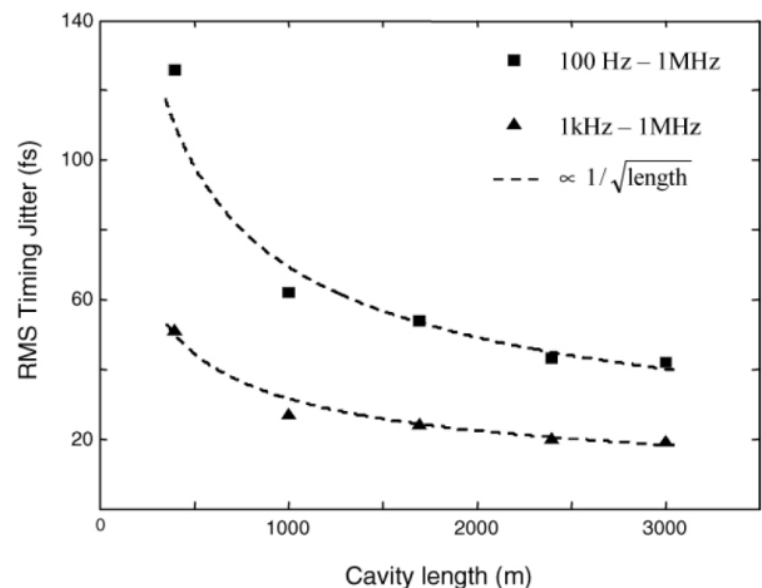

Fig. 4. This graph shows relation between cavity length and timing jitter. The measurement was taken for different fiber lengths length and offset frequencies. The timing jitter drops significantly with increased length of fiber. In both observations jitter is minimized for 3 kilometers fiber length (Lasri et al. 2003). Reprinted with permission of The Optical Society

\section{Multi-loop OEO (MOEO)}

The optical fiber is a key energy storage component in the OEO. It contributes to the high Q in the system. However, as the length of the fiber increases the mode spacing decreases which results in a greater number of modes inside the bandwidth of the filter. As the modes are brought close together, mode competition leads to high frequency noise (Jiang et al. 2013). One solution to this problem is the multi-loop OEO.
Figure 5a shows a schematic diagram of a MOEO. Modulated laser light is optically amplified and passed through two different fiber paths that are arranged in parallel with one another. These signals are recombined and passed, respectively, through a photo-detector and a filter. The amplified signal is then fed back into the modulator. The main difference between the MOEO compared to the SOEO is use of two or more fiber loops in parallel which act as a filter. As shown in figure 5b, the frequency separation of the modes of the short loop is much larger than that for the long loop. When taken together, the oscillation frequency will be determined by the frequency range which is low loss in both modes. Here, the tuning range and tuning resolution is, respectively, determined by the shorter and the longer loop (Yao 2000). For example, with loop lengths of 200 meters and 4 kilometers will result in a mode spacing of 1 $\mathrm{MHz}$ and $50 \mathrm{KHz}$. If we make a MOEO having optical fibers of 200 meters and 4 kilometers in parallel, there will be only a single mode in the frequency range of the shorter loop (1 MHz). That means 20 modes of the longer loop get suppressed by the shorter loop before passing through the band pass filter. This way, large number of parasitic modes can be removed from the output. The oscillation frequency of this configuration must satisfy, $f_{\text {osc }}=\frac{l+\frac{1}{2}}{\tau_{1}}=\frac{m+\frac{1}{2}}{\tau_{2}}$ for $\mathrm{G}\left(\mathrm{V}_{\text {osc }}\right)<0, f_{\text {osc }}=\frac{l}{\tau_{1}}=\frac{m}{\tau_{2}}$ for $\mathrm{G}\left(\mathrm{V}_{\mathrm{osc}}\right)>0$, where $l$ and $m$ are integers, $\tau_{1}$ and $\tau_{2}$ are delay time of the longer and shorter fiber loops respectively (Yao et al. 1998).

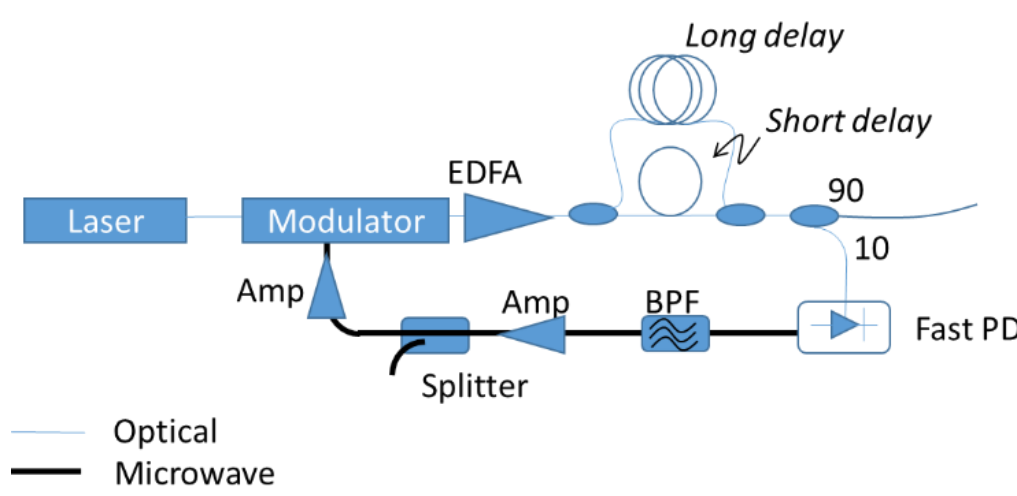

a

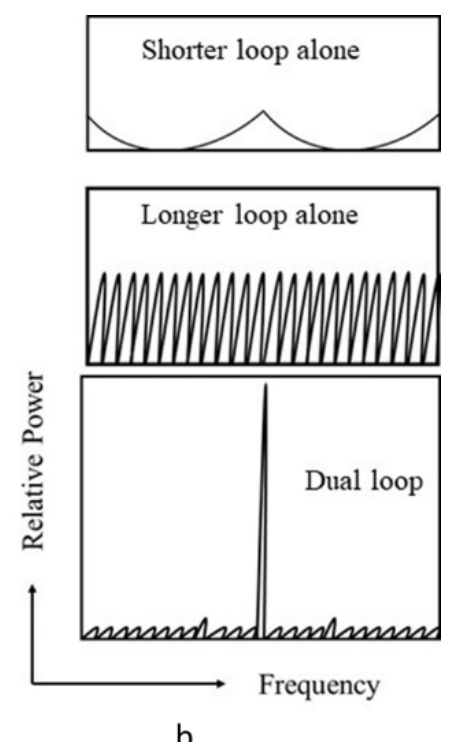

Fig. 5. (a) This is a schematic diagram of a multi-loop OEO. In this configuration, two loops (shorter and longer) are place parallel to each other. (b) The long fiber will have larger number of modes than short one within same bandwidth. When place them in parallel one mode will survive out of many competing modes (Yao 2000) 
Over the years, several variations of MOEOs have been demonstrated. Some of these setups include the use of three loop lengths in series with a variable optical attenuator (Banky et al. 2006), polarizing beam splitters and couplers (Yang et al. 2007), and fiber diffraction gratings within the loops (Shumakher 2008). Figure 6 shows a comparison between the phase noise of one, two, and three loops/loop OEO setup (Banky et al. 2006). Here, measurements were taken in three different stages. First, the output of a single loop OEO was taken by turning the power of the other two fiber inputs off. A similar method was implemented for the other two measurements. Their observation shows that the three loop OEO has the least phase noise and fewest parasitic modes.

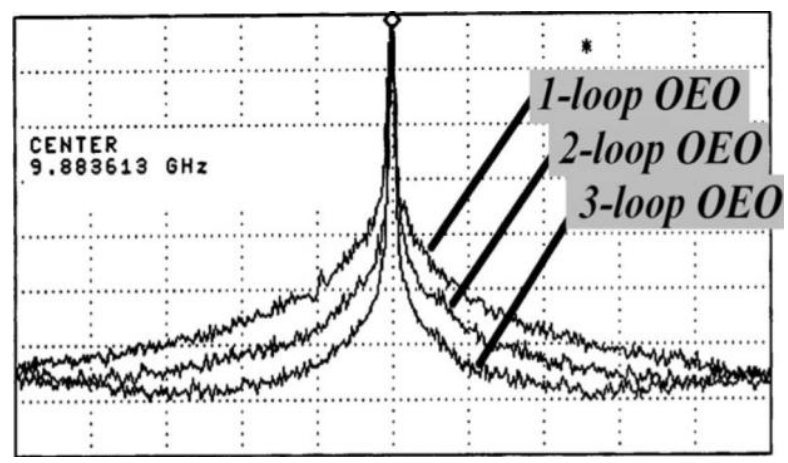

Fig. 6. Comparison between phase noise of one, two, and three multi-loop OEO. The OEO shows improve phase noise performance with increasing numbers of parallel loop delay in SOEO (Banky et al. 2006). Reprinted with permission of The Optical Society

\section{Injection locking OEO (IOEO)}

The reduction of noise from parasitic modes in the output signal is addressed in the MOEO, but the overall $Q$ of the system is reduced because the effective $Q$ is the result of averaging that of the participating loops (Devgan 2013). To improve the effective $\mathrm{Q}$, the injection loop OEO (IOEO) was developed (Yao \& Maleki 1996b), which preserves high $\mathrm{Q}$ and reduced phase noise of the longer loop while the shorter loop provides extended frequency tunability.

The injection loop OEO (IOEO) is a combination OEOs where the RF output from one is injected into the RF portion of the other. In this OEO, higher order output of the longer loop is injected into the shorter loop so that the frequency of the shorter loop is forced to lock with that of the longer loop. This reduces frequency and phase fluctuations. There are two kinds of IOEO: self-injection (SI-OEO) and dual-injection loop (DIL-OEO). The SIOEO is modification of the single loop OEO and is shown in Fig. 7a. In this configuration a longer loop span is connected to the optical output, detected and combined with the electrical section of the primary loop. The injection of a spectrally pure signal into a noisy signal will force latter signal to lock to the former signal (Yao et al. 1998).

Moreover, if we implement few changes in the selfinjection locking OEO we will get a second type of IOEO called a dual-loop injection OEO (DIL-OEO), invented by (Zhou et al. 2005). Only difference between these two types of OEOs is that in the DIL-OEO, the longer loop is not just a loop as in SI-OEO but is also an OEO, as shown in Fig. 7b. In fact, the DIL-OEO is a series combination of two single loop OEOs. The longer loop and the shorter loop OEOs are called primary and secondary OEOs, respectively. Here, the ultra-low phase noise output of the primary OEO is coupled to the secondary OEO and output of the overall system is stabilized. This creative combination of SOEOs has shown better performance against corresponding separate OEOs. For example, if we use a filter with a quality factor on the order of ten thousand and make two separate SOEOs with different fiber lengths, the output of the SOEO with a shorter fiber length will have low resolution: the phase-noise spectral density is inversely proportional to the cavity length (Lasri et al. 2003), whereas the output of the longer loop OEO will be spurious and less tunable: a $4 \mathrm{~km}$ fiber results into $50 \mathrm{KHz}$ mode spacing and a Q-factor of 200,000, such narrow mode spacing cannot be filtered using low Q conventional filters (Zhou \& Blasche 2005). On the other hand, combination of both OEOs will result into ultra-pure, highly stable, and high tunability microwave signal because a longer-loop OEO injectionlocks a short-loop single-mode OEO (Lee et al. 2008, Zou et al. 2016). Little progress on IOEOs has been reported despite its better performance compared with SOEOs. One crucial development was achieved by (Okusaga et al. 2011) wherein they coupled the primary and secondary OEOs in both directions. Jiang et al. (2013), used a higher order modes generator instead of longer loop OEO and injected these modes into an OEO built from a shorter loop. They observed $65 \mathrm{~dB}$ side-mode suppression ratio (SMSR) much better than $18 \mathrm{~dB}$ SMSR in case of freerunning OEO.

\section{OEO with a superconducting band pass filter}

The research discussed thus far in this paper has focused on improving the $\mathrm{Q}$ by increasing the effective loop delay. No very little consideration has been given to optimizing the electrical filter. In most experiments, a filter with a $\mathrm{Q}$ on the order of ten to one-hundred thousand was used (Yao \& Maleki 1996a, Lasri et al. 2003, Eliyahu et al. 2003, Zhou \& Blasche 2005). The transmission bandwidth of the filter is inversely proportional to its Q, so eventually one can no longer increase the loop length without causing mode competition due to the closelyspaced loop modes under the transmission band of the filter. 

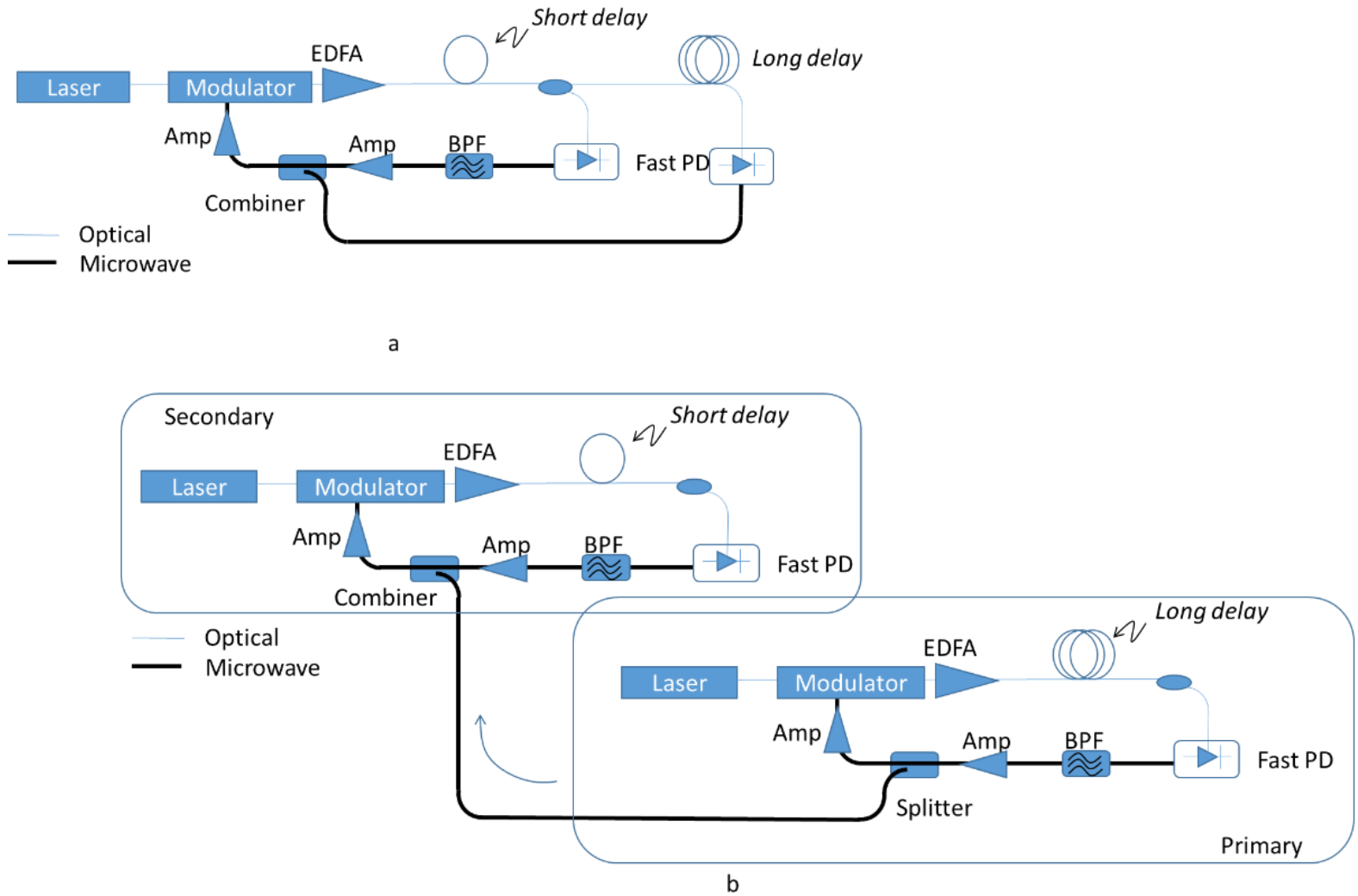

Fig. 7. (a) This is a schematic diagram of a self-injection loop OEO. In this OEO, the optical output of SOEO is detected by a separate photodiode and combined loop with the filtered electrical portion of the OEO (Zhou \& Blasche 2005b). (b) This is a diagram of the dual injection loop OEO. Here the RF output from the primary OEO is injected into the filtered electrical portion of a secondary OEO (Devgan 2013)

The ongoing work in our group at the University of California, Merced involves exploring the impact of high$\mathrm{Q}$ electrical band pass filters. We use quarter-wave stub cavities made from superconducting niobium (Reagor $e t$ al. 2016) in place of the traditional band-pass filter. These SRF cavities can achieve a quality factor on the order of $10^{8}$ or $10^{9}$ with proper fabrication and post-fabrication treatment. Having such a narrow bandwidth filter will allow us to greatly increase the amount of delay which can be added to the optical side of the OEO, while maintaining a reasonable number of optical modes present under the filter envelope. With fewer optical modes being present in the signal after being filtered, along with an efficient method for thermally stabilizing the fiber delay, the output of this OEO should have lower phase noise and timing jitter compared to other implementations. One of our experimental results using an SRF cavity is shown in the Fig. 8.

As expected, the OEO with the longer loop exhibits improved sideband suppression better performance compared with the short loop which has a length of approximately $100 \mathrm{~m}$. Here, niobium band-pass filter of 1 $\mathrm{KHz}$ bandwidth is used. The frequency of SRF cavities can be tuned by translating a dielectric rod into the cavity.

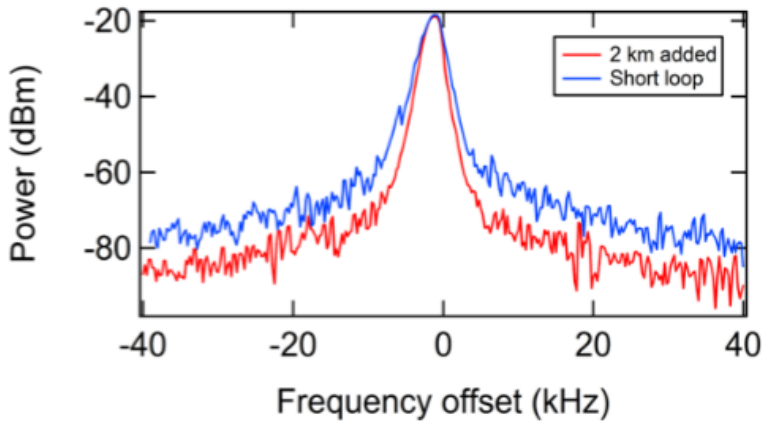

Fig. 8. Electrical output of our OEO set up. The superconducting niobium band-pass filter has $Q$ of 1 billion. The longer loop $(2 \mathrm{Km})$ had shown better phase noise performance compare to the shorter fiber loop (100m)

\section{CONCLUSION}

Since their invention just over 20 years ago the overall performance of OEOs has improved dramatically and the devices have become more practical (HI-Q ${ }^{\mathrm{TM}}$ NANO OEO, OE waves). We expect that the need for practical tabletop frequency sources will drive further development of this type of device. 


\section{ACKNOWLEDGEMENT}

We want to acknowledge Prof. Sharping group for all help and support and Dr. Pashupati Dhakal for his guidance and suggestions.

\section{REFERENCES}

Banky, T., Horváth, B. and Berceli, T. 2006. Optimum configuration of multiloop optoelectronic oscillators. Journal of the Optical Society of America B 23: 1371-1380.

Couch, L. W. 1995. Modern communication systems: principles and applications. Prentice Hall.

Chen, A., and Murphy, E. (Eds.). 2011. Broadband optical modulators: science, technology, and applications. CRC press.

Cui, P., Yang, L., Guo, Y., Lin, J., Liu, Y. and Zhu, J. 2018. Absolute distance measurement using an optical comb and an optoelectronic oscillator. IEEE Photonics Technology Letters 30(8): 744-747.

Devgan, P. 2013. A Review of Optoelectronic Oscillators for High Speed Signal Processing applications. Hindawi Publishing Corporation 2013: 1-16.

Eliyahu, D. Sariri, K., Taylor, J. and Maleki, L. 2003. Optoelectronic oscillator with improved phase noise and frequency stability. SPIE 4998: 139-147.

Eliyahu, D. and Maleki, L. 2003. Low phase noise and spurious level in multi-loop opto-electronic oscillators. In: IEEE Proceedings of International Frequency Control Symposium and PDA Exhibition Jointly with the 17th European Frequency and Time Forum, pp. 405-410.

Eliyahu D., Seidel D. and Maleki L. 2008. Phase noise of a high performance OEO and an ultra low noise floor cross-correlation microwave photonic homodyne system. IEEE International Frequency Symposium: 811-814.

Hajimiri, A. 1998. A general theory of phase noise in electric oscillator. IEEE 33: 179-194.

Hao, T., Cen, Q., Dai, Y., Tang, J., Li, W., Yao, J., Zhu, N. and Li, M. 2018. Breaking the limitation of mode building time in an optoelectronic oscillator. Nature communications 9: 1839.

HI-Q ${ }^{\text {TM }}$ NANO OEO, OEwaves, 465 N. Halstead St, Suite 140 Pasadena, CA 91107.

Jiang, Y., Bai, G., Hu, L., Li, H., Zhou, Z., Xu, J. and Wang, S. 2013. Frequency locked single-mode optoelectronic oscillator by using low frequency RF signal injection. IEEE Photonics Technology Letters 25(4): 382-384.
Kaba, M., Li, H-W., Daryoush, A. S., Vilcot, J-P., Dicoster, D., Chazelas, J., Bouwmans, G., Quiquempois, Y. and Deborgies, F. 2006. Improving thermal stability of opto-electronic oscillators. IEEE microwave magazine 1527-3342: $38-47$.

Lasri, J., Devgan, P., Tang, R. and Kumar, P. 2003. Selfstarting optoelectronic oscillator for generating ultralow-jitter high-rate $(10 \mathrm{GHz}$ or higher) optical pulses. Optics Express 11: 1430-1435.

Lee, K. H., Kim, J. Y. and Choi, W. Y. 2008. Injectionlocked hybrid optoelectronic oscillators for singlemode oscillation. IEEE Photonics Technology Letters 20(19): 1645-1647.

Lesson, D. B. 1966. A simple model of feedback oscillator noise spectrum. Proceeding Letters 54: 329-330.

Liu, A., Dai, J. and Xu, K. 2018. Stable and Low-Spurs Optoelectronic Oscillators: A Review. Applied Sciences 8(12): 2623.

Madjar, A. and Tibor, B. 2006. Microwave generation by optical techniques-A review. $36^{\text {th }}$ European Microwave Conference, IEEE, pp. 1099-1102.

Maleki, L. 2012. The opto-electronic oscillator (OEO): Review and recent progress. In: IEEE proceedings of European Frequency and Time Forum, pp. 497500 .

Marshall, W. K., Crosignani, B. and Yariv, A. 2000. Laser phase noise to intensity noise conversion by lowestorder group-velocity dispersion in optical fiber: exact theory. Optics letters 25(3): 165-167.

Marshall, W. K. and Yariv, A. 2000. Spectrum of the intensity of modulated noisy light after propagation in dispersive fiber. IEEE Photonics Technology Letters 12(3): 302-304.

Merrer, P.H., Brahimi, H. and Llopis, O. 2008. Optical techniques for microwave frequency stabilization: resonant versus delay line approaches and related modelling problems. In: IEEE proceedings of International Topical Meeting on Microwave Photonics jointly held with the Asia-Pacific Microwave Photonics Conference, pp. 146-149.

Nelson, C. W., Hati, A., Howe, D. A. and Zhou, W. 2007. Microwave optoelectronic oscillator with optical gain. In: IEEE proceedings of International Frequency Control Symposium Joint with the 21st European Frequency and Time Forum, pp. 10141019. 
Neyer, A. and Voges E. 1981. Nonlinear electrooptic oscillator using an integrated interferometer. Optics Communications 37: 169-174.

Okusaga, O., Adles, E.J., Levy, E.C., Zhou, W., Carter, G.M., Menyuk, C.R. and Horowitz, M. 2011. Spurious mode reduction in dual injection-locked optoelectronic oscillators. Optic Express 19: 58395854.

Reagor, M., Pfaff, W., Axline, C., Heeres, R.W., Ofek, N., Sliwa, K., Holland, E., Wang, C., Blumoff, J., Chou, K. and Hatridge, M.J. 2016. Quantum memory with millisecond coherence in circuit QED. Physical Review B 94(1): 014506.

Rutman, J. 1978. Characterization of phase and frequency instabilities in precision frequency sources: fifteen years of progress. In: Proceeding of the IEEE, pp. 1048-1075.

Romisch, S., Kitching, J., Ferre-Pikal, E., Hollberg, L. and Walls, F. L. 2000. Performance evaluation of an optoelectronic oscillator. IEEE transactions on ultrasonic, ferroelectrics, and frequency controls 47(5): 1159-1165.

Romisch, S. and De Marchi, A. 1999. Noise predictions for optoelectronic oscillators using different models. In: IEEE Proceedings of the 1999 Joint Meeting of the European Frequency and Time Forum and the IEEE International Frequency Control Symposium, pp. 1100-1104.

Rubiola, E., Salik, E., Yu, N. and Maleki, L. 2006. Flicker noise in high-speed pin photodiodes. IEEE Transactions on Microwave Theory and Techniques 54(2): 816-820.

Salik, E., Yu, N. and Maleki, L. 2007. An ultralow phase noise coupled optoelectronic oscillator. IEEE Photonics Technology Letters 19(6): 444-446.

Shelby, R. M. Levenson, M. D., and Bayer, P. W. 1985. Guided acoustic-wave Brillouin scattering. Physical Review B 31(8): 5244.
Shumakher, E. 2008. A Novel multiloop optoelectronic oscillator. Photonics Technology Letters 20:18811883.

Vorreau, P. 2010. An optical grooming switch for highspeed traffic aggregation in time, space and wavelength (Vol. 6). KIT Scientific Publishing.

Yao, X. S. 2000. Multiloop optoelectronic oscillator. Journal of Quantum electronics 36: 79-84.

Yao, X. S. and Maleki, L. 1996a. Optoelectronic microwave oscillator. Journal of the Optical Society of America B13: 1725-1735.

Yao, X. S., Maleki, L. 1996b. Optoelectronic oscillator for photonic systems. IEEE Journal of quantum electronics 32: 1141-1149.

Yao, X. S., Maleki, L., Ji, Y., Lutes, G, and Tu, M. 1998. Dual-loop opto-electronic oscillator. In: IEEE Proceedings of the 1998 IEEE International Frequency Control Symposium, pp. 545-549.

Yao, X. S., Maleki, L. and Eliyahu, D. 2004. Progress in the opto-electronic oscillator-a ten year anniversary review. In: IEEE 1 Proceedings of the 2004 IEEE MTT-S International Microwave Symposium Digest, pp. 287-290.

Yang, J., Jin-Long, Y., Yao-Tian, W., Li-Tai, Z. and EnZe, Y. 2007. An Optical Domain Combined DualLoop Optoelectronic Oscillator. IEEE Photonics Technology Letters 19: 807-809.

Zhou, W. and Blasche, G. 2005. Injection-locked dual opto-electronic oscillator with ultra-low phase noise and ultra-low spurious level. IEEE Transactions on Microwave Theory and Techniques 53: 929-933.

Zou, X., Liu, X., Li, W., Li, P., Pan, W., Yan, L. and Shao, L. 2016. Optoelectronic oscillators (OEOs) to sensing, measurement, and detection. IEEE Journal of Quantum Electronics 52(1): 1-16. 